\title{
A influência do comportamento parental na adaptação da criança ao atendimento odontológico
}

\author{
The influence of parental behavior on the \\ child's adaptation to dental care
}

\section{Roberta Ferreira Silva' (D) Iza Alves Peixoto ${ }^{2}$}

1'Autora para correspondência. Escola Bahiana de Medicina e Saúde Pública (Salvador). Bahia, Brasil. robertaluciafs@hotmail.com Escola Bahiana de Medicina e Saúde Pública (Salvador). Bahia, Brasil. peixotoiza@gmail.com

RESUMO | A influência do comportamento parental nas crianças produz estímulos aversivos através de relatos de experiências pessoais negativas, por vezes assustadoras, sobre tratamentos dentários traumáticos, medo produzido pelos pais através de ameaças verbais, ou seja, a visita ao dentista citada como uma ameaça, ou como forma de punição. Este trabalho tem como objetivo compreender, através de uma revisão da literatura, a influência de padrões de comportamento dos responsáveis e seu reflexo na adaptação da criança diante do tratamento odontológico. Dessa forma, os objetivos específicos são: 1) compreender a influência do comportamento parental no paciente infantil frente ao atendimento odontológico; 2 ) discorrer sobre os fatores que podem impactar nos níveis de ansiedade do paciente infantil diante da consulta ou tratamento odontológico. A revisão de literatura foi realizada a partir da busca de artigos científicos publicados em periódicos nacionais e internacionais, como PubMed, Scielo, BVS, além de consulta bibliográfica. Os descritores utilizados como parâmetros de pesquisa foram ansiedade, comportamento, influência parental, odontopediatria e tratamento. Os artigos foram selecionados através da análise dos resumos, que tinham como assunto principal os fatores associados à ansiedade odontológica e ao atendimento em Odontopediatria. Espera-se compreender os fatores externos que causam comportamentos de medo e ansiedade no paciente infantil, inclusive o próprio comportamento ansioso da família e os padrões disfuncionais que impactam na evolução do tratamento odontológico, bem como as técnicas e métodos de acolhimento e acomodação utilizada pelos Odontopediatras, a fim de viabilizar o levantamento de hipóteses para possíveis intervenções.

PALAVRAS-CHAVE: Poder familiar. Adaptação psicológica. Odontopediatria. Terapêutica. Ansiedade.
ABSTRACT | The influence of parental anxiety on children produces aversive stimulus through negative personal reports, sometimes frightening, about traumatic dental treatments, fear produced by parents through verbal threats, in other words, the visit to the dentist cited as a threat, or as form of punishment. This assignment aims to understand, through bibliographical research and literature review, the influence of behavioral patterns of parents or guardians and it's reflection on the accommodation and adaptation of the child to dental treatment. Thus, the specific objectives are: 1) to understand the influence of parental behavior on the infant patient in the face of dental care; 2 ) talk about the factors that can impact the levels of anxiety of the infant patient in the face of dental consultation or treatment. The literature review was made based on the search of scientific articles published in national and international journals, such as PubMed, Scielo, BVS, and bibliographic researches. The descriptors used as search parameters were anxiety, behavior, parental influence, pediatric dentistry and treatment. The articles were selected through the analysis of the summaries, which had as main subject the factors associated with dental anxiety and dental care. It's expected to understand the external factors that cause fear and anxiety behaviors in the children as a patient, including the family's own anxious behavior and the dysfunctional patterns that affects the evolution of dental treatment, as well as the techniques and methods of reception and accommodation used by professionals pediatric dentistry, in order to enable the hypothesis for possible interventions.

KEYWORDS: Parenting. Adaptation, psychological. Pediatric dentistry. Therapeutic. Anxiety. 


\section{Introdução}

Mesmo com os avanços tecnológicos e a busca constante pela humanização nos atendimentos de atenção à saúde, a ansiedade entre os pacientes pediátricos durante o tratamento odontológico se apresenta como um dos grandes desafios enfrentados pelo Odontopediatra.

A ansiedade dental, ou medo do dentista, não é uma característica inata dos indivíduos, porém, com o início do processo de socialização na infância, a criança recebe de seus cuidadores, parentes e colegas, estímulos aversivos através de relatos de experiências pessoais negativas, por vezes assustadoras, sobre tratamentos dentários traumáticos. Para, além disso, alguns cuidadores utilizam a visita ao dentista como forma de punir a criança ${ }^{1}$.

Existem múltiplos fatores etiológicos que influenciam no desenvolvimento da ansiedade no paciente odontopediátrico, tais quais os fatores psicológicos, comportamentais e emocionais, a exemplo de algumas características das crianças como timidez e emoção exacerbada 2 .

O comportamento parental também exerce influência no comportamento infantil. Existe uma correlação proporcional entre os níveis de ansiedade infantil e a característica da atitude materna. Desse modo, serão observados menores índices de ansiedade infantil em crianças cujas mães são responsivas, que impõem limites e fazem intervenções - com reforço e punição -, enquanto as crianças cujas mães são mais permissivas, com pouca autoconfiança e que evitam punições ou limitações, apresentam maiores índices de ansiedade ${ }^{3,4}$.

Outro importante aspecto que contribui para que se desenvolva tal condição é a visita tardia ao consultório odontológico, o que tem como agravante a instalação da sintomatologia dolorosa, por meio de cáries, fraturas dentárias ou outras patologias bucais²

É importante que o Odontopediatra esteja preparado para perceber a existência de fatores que possam ocasionar comportamentos de não colaboração diante do tratamento proposto, conhecendo os preditores de comportamento infantil, pois irá facilitar a rotina de atendimento, permitindo que o profissional atue de maneira mais assertiva diante de situações adversas, sem torná-las traumáticas ou provocadoras de sofrimento ${ }^{5}$.
Esse trabalho tem como objetivo compreender, através de uma revisão da literatura, a influência de padrões de comportamento dos responsáveis e seu reflexo na adaptação da criança diante do tratamento odontológico. Dessa forma, os objetivos específicos são: 1) compreender a influência do comportamento parental no paciente infantil frente ao atendimento odontológico; 2) discorrer sobre os fatores que podem impactar nos níveis de ansiedade do paciente infantil diante da consulta ou tratamento odontológico.

\section{Metodologia}

A revisão de literatura foi realizada a partir da busca de artigos científicos nas seguintes bases de dados: PubMed, Scielo, BVS e consulta bibliográfica. Os descritores utilizados como parâmetros de pesquisa foram: poder família (parenting), adaptação psicológica (adaptation psychological), odontopediatria (pediatric dentistry), terapêutica (therapeutic) e ansiedade (anxiety). Os critérios de inclusão foram artigos publicados entre os anos de 2010 e 2019, nos idiomas inglês e português. Os artigos foram selecionados através da análise dos títulos e resumos, que tinham como assunto principal os fatores associados à ansiedade odontológica e ao atendimento em Odontopediatria. O total de referências obtidas foi de 19 artigos. Os critérios de exclusão foram artigos que não possuíam associação direta com o tema abordado. Os artigos que são anteriores a esse período foram anexados devido à importância das informações, selecionado através da análise do resumo.

\section{Revisão de literatura}

\section{A ansiedade infantil no tratamento odontológico}

Quando se fala de tratamento odontológico em crianças, logo vêm os questionamentos sobre o quão difícil este pode ser. De fato, a prática terapêutica com crianças exige esforços e entendimentos que vão além do consultório odontológico. Requer um cuidado com "o todo", considerando o contexto em que vivem, relação com a família, aspectos psicológicos, condições socioeconômicas, entre outros, para se obter conhecimento da história e das vivências do paciente, nos propiciando a construção de uma boa convivência e parceria com os pais ou responsáveis, além da própria criança atendida ${ }^{6}$. 
A relação entre o paciente e o profissional de saúde é um fator preponderante para o sucesso terapêutico, sendo este um diferencial significativo entre o atendimento a crianças e a pessoas adultas. $\mathrm{O}$ tratamento com adultos é pautado na confiança e no estreitamento de laços entre o dentista e seu paciente, o que caracteriza uma relação bilateral. Porém, o tratamento com o paciente infantil requer uma relação triangulada, com participação contínua dos pais ou responsáveis, do profissional e da própria criança ${ }^{6}$.

Considerando as práticas psicológicas atuais, nota-se uma ação conjunta por parte dos psicólogos em relação aos pais e cuidadores das crianças. Tal parceria se torna alicerce para construção da aliança terapêutica que vai proporcionar o sucesso do tratamento. $\mathrm{Na}$ Odontopediatria, a lógica é a mesma, ao tratar da promoção de saúde infantil, prevenção de doenças ou da adesão ao tratamento, é necessário que o Odontopediatra envolva os pais e responsáveis, orientando-os acerca do seu papel, visando atingir objetivos que beneficiem a criança ${ }^{3}$.

Segundo Kilinç et al.?, algumas crianças apresentam medo e ansiedade quando são expostas a visitas ao dentista e existem fatores específicos que podem explicar tal comportamento. Na Odontologia, esses fatores consistem nos instrumentos odontológicos, que ainda são desconhecidos para o paciente pediátrico, nos métodos de tratamento, no medo e/ou na dor decorrente de tratamentos anteriores, nos prejuízos resultantes da primeira visita tardia ao dentista, na presença de funcionários da clínica, que são pessoas estranhas para elas e no medo de outras experiências vividas, não necessariamente com tratamento dental, mas através de outros procedimentos médicos ${ }^{7}$.

Segundo Venham, Bengston e Cipes ${ }^{8}$, o comportamento das crianças tende a melhorar nas consultas odontológicas subsequentes, sugerindo que essas visitas ajudam a criança a se familiarizar com o ambiente e com os procedimentos odontológicos mais estressantes. Apesar dos esforços para adequar os equipamentos odontológicos, os consultórios e até mesmo os EPIs, como jalecos, máscaras e gorros, adotando uma aparência mais leve e acolhedora para o paciente infantil, ainda é distante imaginar que a prática odontológica perca sua característica invasiva, decorrente da localização da área de atuação do cirurgião-dentista $\%$.
Para um melhor entendimento e clareza dos termos empregados neste trabalho, cabe ressaltar os conceitos de ansiedade e medo, bem como salientar diferença entre essas palavras. A ansiedade "consiste num estado emocional inevitável, mas que se torna persistente e desagradável a quem sente". O nível da dor tem relação diretamente proporcional ao nível de ansiedade, segundo Félix et al. ‥ Já o medo "é uma sensação que evoca um estado de alerta que se manifesta pelo receio de fazer alguma coisa, geralmente por se sentir ameaçado física ou psicologicamente". Ainda segundo Félix et al. 9 , o medo pode ser classificado como direto e indireto, sendo que o primeiro é percebido a partir da própria vivência do sujeito, enquanto o segundo é adquirido a partir de relatos de terceiros, ou seja, situações que foram vivenciadas por outras pessoas e não pelo próprio indivíduo?

Shind e Hedge ${ }^{10}$ indicaram a "ansiedade dentária" como o sentimento ou estado apreensivo decorrente do tratamento dentário, que não precisa estar via de regra, relacionado a um estímulo específico. Já o "medo odontológico", para os autores, se refere a uma reação emocional normal a, pelo menos, um estímulo que represente ameaça face ao contexto odontológico ${ }^{10}$.

\section{Conhecimento sobre ansiedade e as contribuições da Psicologia}

Diante das evidências e estudos desenvolvidos sobre o comportamento ansioso na infância, faz-se necessário a compreensão dos profissionais de Odontopediatria acerca dos processos de desenvolvimento da ansiedade em crianças. Friman Hayes e Wilson indicaram uma imprecisão na abordagem psicológica para o termo ansiedade, de modo que é empregado em diferentes sistemas explicativos e eventos diversos. $\mathrm{O}$ uso de metáforas, segundo os autores, dificulta a construção de definições consistentes dos fenômenos para os quais os cientistas se voltam ${ }^{11}$.

Segundo Petersen ${ }^{12}$ as emoções, comportamento, pensamentos e o corpo são as dimensões nas quais a ansiedade é expressa. Pensando num contexto de abordagem da Terapia Cognitivo-Comportamental (TCC), a intervenção se direciona para as dimensões comportamental, cognitiva, emocional e social. "Práticas, recompensas, tarefas para casa, observação do processamento da informação, nomeação de sentimentos e participação dos pais e pares" são as técnicas utilizadas no contexto do tratamento12. 
Setiawan, Agustiani e Kendhawati $i^{13}$ apresentaram evidências do campo da psicologia comportamental para explicar o desenvolvimento da ansiedade infantil relativa ao tratamento odontológico. Para eles, o medo e a ansiedade são vistos como estímulos condicionados transmitidos dos pais para os filhos, e que combinado com estimulo aversivo incondicionado (o próprio tratamento odontológico) "expressam um medo condicionado em relação ao dentista". Isto ocorre, pois assimilam o tratamento como algo ruim e doloroso $\frac{13}{3}$.

Para Silva et al. $\frac{14}{} \mathrm{o}$ aprendizado pode acontecer por meio da observação de terceiros. Desse modo, o indivíduo pode ter um comportamento reforçado por experiências indiretas, desenvolvendo respostas a partir da aprendizagem vicária.

Martins e Dias ${ }^{15}$ sugerem que as crianças aprendem, também, através da observação do ambiente onde vivem, trata-se de uma aprendizagem adaptativa e geralmente imitam seus familiares e indivíduos de sua convivência, desta forma internalizam seus hábitos e, a partir desta vivencia de medo e ansiedade, a criança pode ser induzida a preconcepção de que ir ao dentista é uma experiência ruim, dolorosa. $\mathrm{O}$ contágio emocional envolve uma tendência à imitação e sincronização automática de expressões, posturas, vocalizações e movimentos com o outro, em um ciclo emocional ${ }^{15}$.

As pesquisas sobre essa temática indicam maior ocorrência de ansiedade consequente entre mães e crianças, principalmente na idade pré-escolar, quando a dependência parental é, naturalmente, mais acentuada ${ }^{13}$.

Apesar da vasta existência de pesquisas científicas que se debruçam sobre a ansiedade no contexto Odontopediátrico, nota-se ainda a carência de uma interação maior entre os diferentes campos do conhecimento para obtenção de meios mais adequados de enfrentamento do problema em face. Ainda que existam referências a autores da Psicologia na abordagem desse tema, ainda não são observadas com frequência atuações conjuntas de profissionais das áreas correlatas para viabilização do tratamento Odontopediátrico, bem como da ansiedade infantil.

\section{O papel da família no desenvolvimento da ansiedade infantil}

A ansiedade odontológica em crianças tem como principal componente a chamada ansiedade parental, que é transmitida das mães, pais ou familiares para as crianças, através de relatos de experiências dentárias traumáticas, descrições de tratamentos invasivos, como extrações ou tratamentos endodônticos, medo produzido pelos pais através de ameaças verbais, ou seja, a visita ao dentista citada como uma ameaça, ou como forma de punição, para levar a criança a fazer suas atividades diárias, como escovar os dentes ${ }^{13}$.

Alguns pais adotam ainda um discurso que faz o dentista parecer mau, através de afirmações como "se você não escovar os dentes, vou te levar ao dentista". Caso a criança apresente resistência durante a consulta, podem haver ainda as punições físicas, que tendem a resultar em trauma para a criança ${ }^{13}$. O comportamento da criança no ato da consulta, inclusive sua disposição colaborativa, será influenciado também pelos antecedentes aos quais ela está exposta, de modo que eventos traumáticos na história individual acarretam maior probabilidade de estímulos aversivos no ato da consulta ${ }^{3}$.

Venham, Murray e Gaulin-Kremer ${ }^{4}$ apontaram ainda uma correlação entre a atitude das mães em relação aos seus filhos e o nível de ansiedade apresentado pelas crianças. Os autores observaram que as mães mais ativas, impositivas e interventivas, exercendo controle sobre o comportamento infantil por meio de reforço e/ou punição, conseguem lograr maior êxito quanto ao controle do comportamento ansioso dos filhos. De igual modo, as crianças cujas mães são mais permissivas, com pouca autoconfiança e que evitam punições ou limitações, apresentam maior incidência de comportamento ansioso durante as consultas e intervenções Odontológicas

Os autores fazem indicação da figura materna como principal, por vezes única, responsável pelo acompanhamento das crianças em suas atividades diárias, bem como pelos cuidados da saúde dos filhos e, por consequência, responsável pelo desenvolvimento da ansiedade infantil. Os termos "mãe" e/ou "materna (o)", aparecem diretamente relacionados aos quadros de ansiedade infantil. Em observância a isso e às mudanças de paradigmas sociais que discutem os 
papeis de homens e mulheres no contexto sociocultural, foi adotado neste trabalho o termo "parental", a fim de abarcar todos os responsáveis envolvidos nos cuidados e educação da criança.

Cassoni ${ }^{16}$ e Cruz et al. ${ }^{17}$ reforçam a relação direta entre o contexto familiar, sobretudo os parâmetros educacionais exercidos, e o desenvolvimento de comportamentos disfuncionais - a exemplo do comportamento ansioso - e os reflexos ao tratamento odontopediátrico. Cassoni ${ }^{16}$ se refere aos estilos parentais para caracterizar os padrões de relacionamento construídos entre pais e filhos, fazendo uma análise proporcional, a partir dos estudos de Weber, Baumrind, Maccoby e Martin, entre responsividade e exigência dos pais em relação aos seus filhos, sendo a exigência as atitudes de controle e a responsividade as ações compreensivas ${ }^{16}$. Cruz et al. ${ }^{17}$ indicam uma atenção ao equilíbrio entre esses dois fatores, uma vez que, de acordo com os autores a exigência em excesso pode ocasionar em ultra disciplina, frustração, complexidade e angústia, enquanto que o relaxamento demasiado tende a comportamentos de dominação, indisciplina, egoísmo, insegurança e dependência ${ }^{16,17}$.

Desse modo, os autores definem os estilos parentais como negligente, indulgente, autoritário e autoritativo. Pais com comportamento negligente são aqueles que apresentam baixos níveis de exigência e responsividade resultando em baixa demonstração de afetividade e exigência. Como consequência desse comportamento paternal as crianças tendem a apresentar submissão ou agressividade. Os pais com estilos de comportamento indulgente apresentam uma combinação de baixa exigência com alta responsividade. A consequência desse comportamento é o excesso de tolerância por parte dos pais resultando em falta de delimitação de regras e limites. Com poucas demandas de responsabilidade e maturidade, o que pode ser observado durante a consulta odontológica é uma criança infantilizada, mimada, egoísta, birrenta, insegura, dependente e por vezes dominadora. Eventualmente esse comportamento da criança pode levar ao abandono do tratamento odontológico $\frac{16,17}{17}$.

O comportamento autoritário dos pais, por sua vez, é marcado por altos níveis de controle e baixa responsividade. Esses pais impõem altos níveis de exigência, estabelecendo regras restritivas e exigências exageradas. Como consequência dessas atitudes, observamos crianças ultra disciplinadas, com presença de frustação, complexidade e angustia. Durante o atendimento odontológico as crianças tendem a ser bem comportadas, porem devido ao medo exacerbado não conseguem estabelecer dialogo e podem não verbalizar dores ${ }^{16,17}$.

Nos três comportamentos paternais apresentados, o desequilíbrio entre os níveis de exigência e reponsividade são fatores preponderantes para o desenvolvimento de problemas emocionais e comportamentais, portanto a ansiedade da criança no tratamento odontológico é bastante presente. Já os pais com comportamento autoritativo apresentam um equilíbrio entre exigência e responsividade. Esses pais monitoram a conduta dos filhos direcionando o comportamento com correções de postura negativas e reforços de posturas positivas, baseando suas ações no respeito mútuo. O reflexo desse comportamento paterno nas crianças é o desenvolvimento das habilidades sociais e aumento da maturidade resultando em indivíduos seguros, afetuosos, com baixos níveis de ansiedade, disciplinados e com boa autoestima. No atendimento odontológico são participativos, colaborativos e apresentam comportamento positivo frente ao tratamento proposto ${ }^{16,17}$.

Outro aspecto importante que se deve observar sobre o comportamento das crianças são os desajustes familiares. Segundo Corrêa, Pinto e Echeverria ${ }^{6}$, crianças cujas famílias apresentam desajustes estruturais contínuos e crônicos, podem ter mais dificuldades durante o tratamento odontológico. Estes desajustes podem ser caracterizados por ocorrências constantes de brigas entre membros da família, divórcio, ou mesmo excesso de ocupação dos pais, seja por problemas de trabalho, vaidades pessoais ou até mesmo o nascimento de um (a) irmão (ã). Expostas a este contexto, as crianças normalmente demonstram insegurança, irritação, choro e certa ansiedade em decorrência da falta de estruturação familiar, por isso o profissional deve estar atento ao comportamento do paciente Odontopediátrico, devendo questionar os responsáveis por eventuais acontecimentos desagradáveis e que possam comprometer a estabilidade emocional da criança ${ }^{6}$.

Diante dos fatores externos que impactam os comportamentos de medo e ansiedade apresentados pelo paciente infantil, incluindo o próprio comportamento ansioso da família, o Odontopediatra deve investir na orientação, direcionando as atitudes dos pais no sentido de contribuir para a evolução do tratamento odontológico, sendo indispensável que haja 
uma concordância de pensamentos e ações que permitam que o tratamento da criança seja realizado de forma respeitosa e humana ${ }^{18}$.

Os autores reforçam a importância da anamnese, que inclui também informações de tratamento pessoal, o que possibilita maior acomodação do paciente ${ }^{18}$. As orientações contemplam, também, a naturalidade que os pais devem ter com as possíveis reações do filho, seja de choro, medo ou até mesmo de hostilidade e violência, de modo que também é importante a compreensão com as técnicas de manejo adotadas pelo profissional, que pode envolver ainda um tom de voz mais firme ou mesmo a retirada dos acompanhantes do ambiente ${ }^{6}$.

Os pais devem contribuir para naturalização da relação com o profissional, desde a explicação honesta do que é a visita ao dentista, até a valorização da curiosidade, da interação com o dentista, até que esteja estabelecida uma relação de confiança entre os envolvidos ${ }^{6}$.

As demandas dos pais/responsáveis também são tratadas pelos autores, de modo que os temores e inquietações dos adultos sejam controlados, tanto no que diz respeito a não permitir que a criança tenha acesso a histórias que potencializem sua ansiedade ou medo do dentista, quanto ao solicitar a não intervenção dos acompanhantes durante $o$ atendimento, sugerindo uma interação mínima com a criança naquele momento ${ }^{6}$.

\section{Contribuições da odontopediatria}

Silva et al. ${ }^{14}$ defendem que o profissional deve conversar com os pais sobre técnicas de manejo comportamental que são utilizados nos atendimentos Odontopediátricos, as quais viabilizam o tratamento por prover ao paciente segurança e confiança no trabalho do profissional. O manejo comportamental no atendimento odontológico a crianças tende a minimizar possíveis riscos de acidentes durante os procedimentos, garantindo a qualidade do serviço. As técnicas descritas pelos autores são de comunicação verbal, comunicação não verbal, dizer-mostrar-fazer, controle de voz, distração, reforço positivo e modelo ${ }^{14}$.

\section{Técnica de comunicação verbal}

Consiste na explicação, dizer ao paciente cada procedimento a ser realizado durante o seu tratamento ${ }^{6}$.

\section{Comunicação não verbal}

Traz o contato, a postura, a expressão facial e a linguagem corporal como reforço e orientação ao paciente sobre o que foi dito verbalmente ${ }^{6}$.

\section{Dizer- mostrar- fazer}

Esta técnica tem o objetivo de reduzir a ansiedade da criança diante de uma situação desconhecida, consiste em apresentar aos poucos à criança alguns elementos do consultório, através de explicações verbais, dos procedimentos odontológicos, utilizando uma linguagem simples, demonstração visual, auditiva e tátil dos mesmos procedimentos que estão sendo realizados ${ }^{18,19}$.

É a técnica mais utilizada na Odontopediatria e consiste em uma explicação ao nível de compreensão da criança do procedimento a ser realizado, seguido da demonstração do que vai ser feito e após esse esclarecimento verbal realizar o procedimento.

\section{Controle de voz}

É uma técnica onde o volume e o tom de voz deverão ser adaptados conforme a necessidade, para se ter a atenção do paciente infantil, a instrução deve ser dada de forma clara e sucinta ${ }^{18}$.

\section{Distração}

Para a técnica de distração da criança podem ser utilizadas músicas, vídeos, histórias infantis e outros recursos audiovisuais através de TVs e tablets. A música, além de relaxar a criança, se sobrepõe ao som dos aparelhos odontológicos. Conversar com a criança sobre outros assuntos e deixar que ela segure algum tipo de brinquedo são métodos que complementam e colaboram para um bom relacionamento entre paciente e profissional18,19. 


\section{Reforço positivo}

Motivação do comportamento positivo da criança, através de elogios, gestos positivos, expressão facial, além dos reforçadores como lembrancinhas e brinquedos 18 .

\section{Modelagem ou imitação}

Consiste na utilização de vídeos e comportamento de outras crianças que já se encontram condicionadas ao tratamento, servindo de modelo para o paciente que está tendo primeiro contato com dentista ou que já tenha alguma experiência não positiva. Dessa forma, proporcionar um novo padrão de comportamento utilizando do aprendizado da criança que é baseado na observação e imitação de outros ${ }^{18}$.

O procedimento pode ser realizado em outro paciente (normalmente mais velho e com bom comportamento) ou bonecos, servindo de modelo, para que encoraje um pouco mais a criança na hora do primeiro contato com o atendimento.

\section{Estabilização protetora}

É uma técnica que restringe os movimentos físicos da criança quando a mesma não colabora com o procedimento. Essa técnica é a última opção a ser usada quando a criança não coopera em nenhuma das outras existentes. Para que seja feita a contenção física precisa de um termo de autorização dos pais.

\section{Orientações aos pais/acompanhantes}

Como forma de facilitar a relação do cirurgião-dentista com as crianças e seus pais/responsáveis que acompanham os atendimentos, Corrêa, Pinto e Echeverria ${ }^{6}$ enumeraram uma série de orientações, baseado na literatura clássica disponível, sobre como os pais/responsáveis podem agir para atenuar o comportamento de ansiedade e inquietação dos pacientes infantis durante o tratamento.

As preocupações apresentadas vão desde os cuidados mais básicos de comportamento pré-sessão até as situações mais complexas e que exijam intervenções mais incisivas do profissional, a fim de garantir o sucesso do tratamento ${ }^{6}$. Neste trabalho destacamos as seguintes orientações: não se aflija com o choro de seu filho; não repreenda o choro da criança; nunca engane a criança, diga-lhe que esta indo ao dentista; não interfira na conversa entre a criança e o odontopediatra; leve seu filho a consultas preventivas para criar o hábito; permita que seu filho se desenvolva sozinho.

\section{Considerações finais}

De um modo geral, a ansiedade no contexto do tratamento Odontopediátrico pode estar relacionada tanto aos fatores inerentes ao próprio ambiente do consultório quanto, principalmente, a eventos antecedentes ligados à relação da criança com seus pais, o ambiente familiar como um todo e os estímulos aos quais ela está exposta.

A ansiedade infantil no contexto do tratamento odontopediátrico está relacionada ao comportamento parental de várias formas. As principais formas identificadas neste trabalho foram o estilo do comportamento dos pais no que diz respeito à educação da criança e informações das experiências pregressas familiares transmitidas às crianças. Outro aspecto identificado abrange os fatores que influenciam nos níveis de ansiedade. Dentre eles, podemos destacar as experiências pregressas da própria criança, a visita tardia ao dentista com manifestações da sintomatologia dolorosa, a associação das visitas e/ou tratamento odontológico como forma de punição e assimilação dos sentimentos de terceiros em relação ao dentista como as que ocorrem nos ambientes escolares e salas de espera dos consultórios.

\section{Contribuições dos autores}

Silva RLF e Peixoto ITA participaram da revisão, desenho metodológico, análise dos artigos, escrita do artigo científico e aprovação da sua versão final.

\section{Conflitos de interesses}

Nenhum conflito financeiro, legal ou político envolvendo terceiros (governo, empresas e fundações privadas, etc.) foi declarado para nenhum aspecto do trabalho submetido (incluindo, mas não se limitando a subvenções e financiamentos, participação em conselho consultivo, desenho de estudo, preparação de manuscrito, análise estatística, etc.). 


\section{Referências}

1. Busato $P$, Garbin RR, Santos CN, Paranhos LR, Rigo L. Influence of martenal anxiety on chil anxiety during dental care: crosssectional study. São Paulo Med J. 2017; 135(2):116-122. http:// dx.doi.org/10.1590/1516-3180.2016.027728102016

2. Soares FC, Lima DSM, Barreto KA, Colares V. A ansiedade odontológica em crianças e os fatores associados: Revisão de literatura. Psic Saúde \& doenças. 2015;16(3):373-385. http:// dx.doi.org/10.15309/15psd160308

3. Brandenburg OJ, Marinho-Casanova ML. A relação mãe-criança durante o atendimento odontológico: Contribuições da análise do comportamento. Estud Psicol. 2013;30(4):269-640. http://dx.doi. org/10.1590/S0103-166X2013000400016

4. Venham LL, Murray P, Gaulin-Kremer E. Child-rearing variables affecting the preschool child's response to dental stress. Clinica Science. 2015;58(1):2042-5. http://dx.doi.org/10.1177/002203457 90580110101

5. Cademartori MG. Comportamento infantil durante consultas odontológicas sequenciais: influência de características clínicas, psicossociais e maternas [dissertação]. Pelotas, RS: Universidade Federal de Pelotas; 2014.

6. Corrêa MSNP, Guedes-Pinto AC, Echeverria S. Influências familiares e conselhos aos pais. In: Guedes-Pinto AC, Mello-Moura ACV. Odontopediatria. Rio de Janeiro: Guanabara Koogan; 2015. p. 125-130.

7. Kilinç G, Akay A, Eden E, Sevinç N, Ellidokuz H. Evaluation of children's dental axiety levels at a kindergarten and at a dental clinic. Braz Oral Res. 2016;30(1):1-8. http://dx.doi.org/10.1177/002 20345790580110101

8. Venham L, Bengston D, Cipes M. Children's response to sequential dental visits. J Dent Res 1977;56(5):454-9. http://dx.doi. org/10.1177/00220345770560050101

9. Felix LF, Brum SC, Barbosa CCN, Barbosa O. Aspectos que influenciam nas reações comportamentais de crianças em consultórios odontológicos. Revista Pró-univerSUS. 2016;7(2):1316.
10. Shind SD, Hegde RJ. Evaluation of parental anxiety on children's behavior and understanding children's dental anxiety after sequential dental visits. Indian J Dent Res. 2017;28(1):22-26. http://dx.doi.org/10.4103/ijdr.IJDR 18116

11. Firman PC, Hayes SC, Wilson KG. Why behavior analysts should study emotion: the exemple of anxiety. J Appl Behav Anal. 1998;31(1):137-156. http://dx.doi.org/10.1901/jaba.1998.31-137

12. Petersen CS. Evidências de efetividade e procedimentos básicos para terapia cognitivo-comportamental para crianças com transtornos de ansiedade. Rev Bras Psicoter. 2011;13(1):39-50.

13. Setiawan AS, Agutiani H, Kendhawati L. Qualitative study on parental behavior as the source of dental fear development as reported by preschool students in Bandung. Eur J Dent. 2018;12(4):480-484. http://dx.doi.org/10.4103/ejd.ejd_258_18

14. Silva JPM. Educação do campo Psicologia da aprendizagem. 1.ed. Santa Maria, RS: Núcleo de Tecnologia Educacional. 2017. 92p.

15. Martins N, Dias MR. Contágio emocional de ansiedade encarregado de educação/criança em Odontopediatria. Rev Port Estomatol Med dent Cir Maxilofac. 2016;57(3):164-170. http:// dx.doi.org/10.1016/j.rpemd.2015.12.006

16. Cassoni C. Estilos parentais e praticas educativas parentais: Revisão sistemática e critica da literatura [dissertação]. Ribeirão Preto, SP: Universidade de São Paulo; 2013.

17. Cruz MVA, Vieira LDS, Ferreira RB. Identificando a criança problema em Odontopediatria: Revisão de literatura. R Odontol Planal Cent. 2018;5(1):10-14.

18. Silva LFP, Freire NC, Santana RS, Miasato JM. Técnicas de manejo comportamental não farmacológicos na Odontopediatria. Rev Odontol Univ Cid São Paulo. 2016; 28(2):135-142.

19. Rocha RASS, Rolim GS, Moraes ABA. Procedimento preparatório para atendimento de pacientes não colaboradores em Odontopediatria. Acta Comportamentalia. 2015; 23(4):423435. 